\title{
A resolution insensitive to geometrical aberrations by using incoherent illumination and interference imaging
}

\author{
Peng Xiao ${ }^{1}$, Mathias Fink ${ }^{1}$, Amir H. Gandjbakhche ${ }^{2}$, and A. Claude Boccara ${ }^{1, a}$ \\ 1 Institut Langevin, ESPCI Paris, PSL Research University, Paris, France \\ 2 National Institute of Child Health and Human Development, National Institutes of \\ Health, Bethesda, MD, USA
}

Received 2 January 2017 / Received in final form 25 January 2017 Published online 25 May 2017

\begin{abstract}
This contribution is another opportunity to acknowledge the influence of Roger Maynard on our research work when he pushed one of us (ACB) to explore the field of waves propagating in complex media rather than limiting ourselves to the wavelength scale of thermal waves or near field phenomena.

Optical tomography is used for imaging in-depth scattering media such as biological tissues. Optical coherence tomography (OCT) plays an important role in imaging biological samples. Coupling OCT with adaptive optics $(\mathrm{AO})$ in order to correct eye aberrations has led to cellular imaging of the retina. By using our approach called Full-Field OCT (FFOCT) we show that, with spatially incoherent illumination, the width of the point-spread function (PSF) that governs the resolution is not affected by aberrations that induce only a reduction of the signal level. We will describe our approach by starting with the PSF experimental data followed by a simple theoretical analysis, and numerical calculations. Finally full images obtained through or inside scattering and aberrating media will be shown.
\end{abstract}

\section{Introduction: OCT and FFOCT background}

Tissue optical imaging suffers from scattering due to the heterogeneous structures inside biological samples. In-depth structures hindered by scattering could be images by many optical imaging techniques that have been developed to be able to select singly back-scattered photons, such as confocal microscopy [1], multiphoton microscopy [2] or optical coherence tomography (OCT) [3]. Scanning OCT selects ballistic photons through scattering media based on spatially coherent and temporally incoherent light sources using an interferometric detection [3]. Point-by-point longitudinal $[4,5]$ and en face scanning $[6,7]$ has been used. Camera coupled OCT systems, which take images that are perpendicular to the microscope optical [8-10], have also been developed use spatially coherent illumination. Higher resolutions are achieved in these systems as

\footnotetext{
a e-mail: claude.boccara@espci.fr
} 


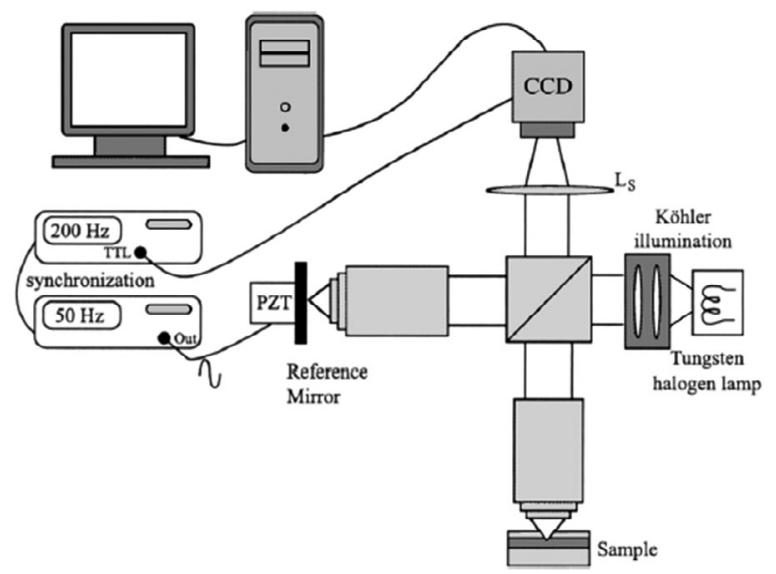

Fig. 1. Schematic FFOCT system [12].

en face acquisition allows using larger numerical aperture optics; but the image can be significantly degraded by coherent cross-talks in these setups [11].

As the schematic shows in Figure 1, a specific en face approach of OCT, Full-Field OCT or FFOCT, was developed in our laboratory [12]. Spatially incoherent broadband light source like halogen lamp or LED is coupled to a Linnik interferometer that has identical microscope objectives in each arm. Optical slices that are perpendicular to the optical axis are selected. They do not require the usual large depth of field, allowing obtaining micron scale resolution in 3D. The cross-talk noises are reduced significantly due to the use of spatially incoherent illumination. The optical beam from the source is divided and recombined by a beamsplitter and imaged on the camera. The FFOCT images formed by interferometric amplitudes are obtained using the combination of two or four phase shifting images by modulating the path difference with the PZT in the reference arm. FFOCT has been working perfectly with ex vivo biological samples and has shown great potential as a non-invasive diagnostic tool for different kind of diseases.

\section{Aberrations fuzziness and PSF}

We are used to observe images that are affected by aberrations (Fig. 2). They are various equivalent ways to quantify the fuzziness either in the real space using the Point Spread Function (PSF) that reflects the spread of the image of a spatial unit source of light in the object plane or its Fourier transform (transfer function) that express the contrast of periodic object images [Born and Wolf].

In absence of aberration the PSF is a diffraction-limited spot. Aberrations are often due to the optical system design (e.g. a photographic lens at full aperture) or the wavefront distortions that happens along the light path from the object to its image. This is the case in astronomy where images of stars are blurred by the atmospheric turbulence or in human vision because of the eye aberrations are more and more pronounced when the pupil size is increase (e.g. night vision). In both case successful aberration corrections have been obtained by using adaptive optics (AO). Indeed $\mathrm{AO}$ is set in order to introduce a wavefront distortion that compensates the aberrations of the atmosphere or of the eye.

In the hypothesis of a linear relationship between the object considered as a source and its image, there is a simple relation between the object, the image and the 

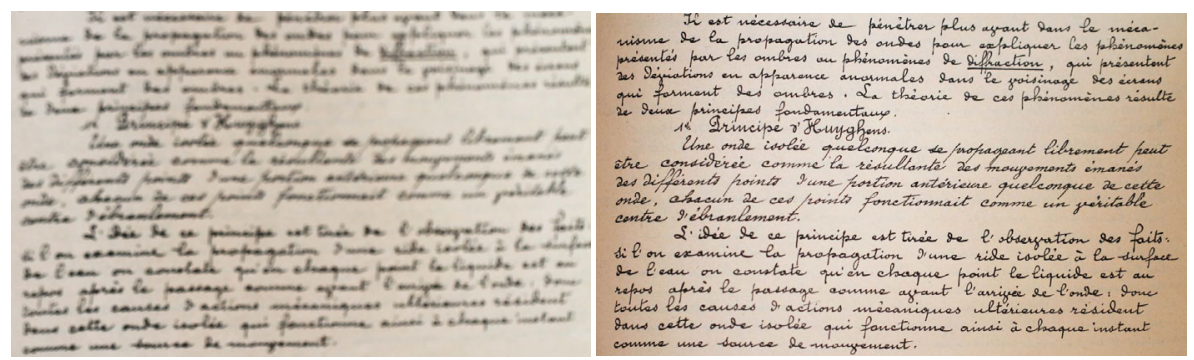

Fig. 2. Aberrations reduce the sharpness of the images (Cornu, Polytechnique, 1897).

PSF: the image is the convolution product of the object (within the magnification of the optical system) and the PSF. The spatial coherence of the object illumination must be considered and complex amplitudes must be used in the case of a coherent illumination, whereas intensities are used for an incoherent illumination. Here we will use coherent as well as incoherent illuminations but because we are dealing with imaging interferometers we will have to consider amplitudes.

\section{The unexpected PSF determination using nanoparticles}

Experiments were first conducted using a commercial FFOCT system LLtech Light-CT scanner [13] with gold nanoparticles in order to follow how the PSF would be affected by various level of aberration. These first experimental results as well as a few numerical simulations have been published in a letter [14]. The PSF of such system has a width of about 1.5 micrometer, meaning that with gold nanoparticles having a diameter much smaller than this value, we can record a reliable PSF. The $40 \mathrm{~nm}$ radius gold particles solution was diluted and dried on a coverslip so that single particles could be imaged. By moving the sample stage by $10 \mathrm{um}, 20 \mathrm{um}$ and $30 \mathrm{um}$ a variable noticeable defocus was induced to the targeted particle if we account for the 0.3 numerical aperture of the microscope. The initial system resolution of $1.5 \mathrm{um}$ corresponds to 2.5 pixels on the camera. By adding $10 \mathrm{um}, 20 \mathrm{um}$ and $30 \mathrm{um}$ defocus, the sample PSF would be broadened by 2.3 times, 4.6 times and 6.9 times. FFOCT images (Figs. 3a-3d) and the corresponding signal profiles (Figs. 3e-3h) of the same nanoparticle were displayed in Figure 3. With more defocus added the signal level of the gold nanoparticles is reduced, but the normalized signal profiles graph (Fig. 3i) shows clearly that the size of the particle that corresponds to the system PSF width keeps the same for all the situations.

One can argue that the PSF is not strictly the same: indeed one can first observe more pronounced wings. As we will see later this is due to the fact that we shift from the product of 2 Bessel functions (Reference PSF times the Object PSF) of the same width (Object PSF without aberrations) to a single Bessel one. The symmetry breaking could be due to a small misalignment of the microscope objective. Finally we have to underline that if the width appears unaffected by the strong defocus the magnitude of the PSF is reduced by more than 20; this signal reduction will be the metric of our aberration correction.

\section{Resolution almost insensitive to aberrations: why?}

As mentioned before aberrations are known to blur optical images by perturbing the wavefronts; more precisely the distorted optical images are obtained by amplitude 

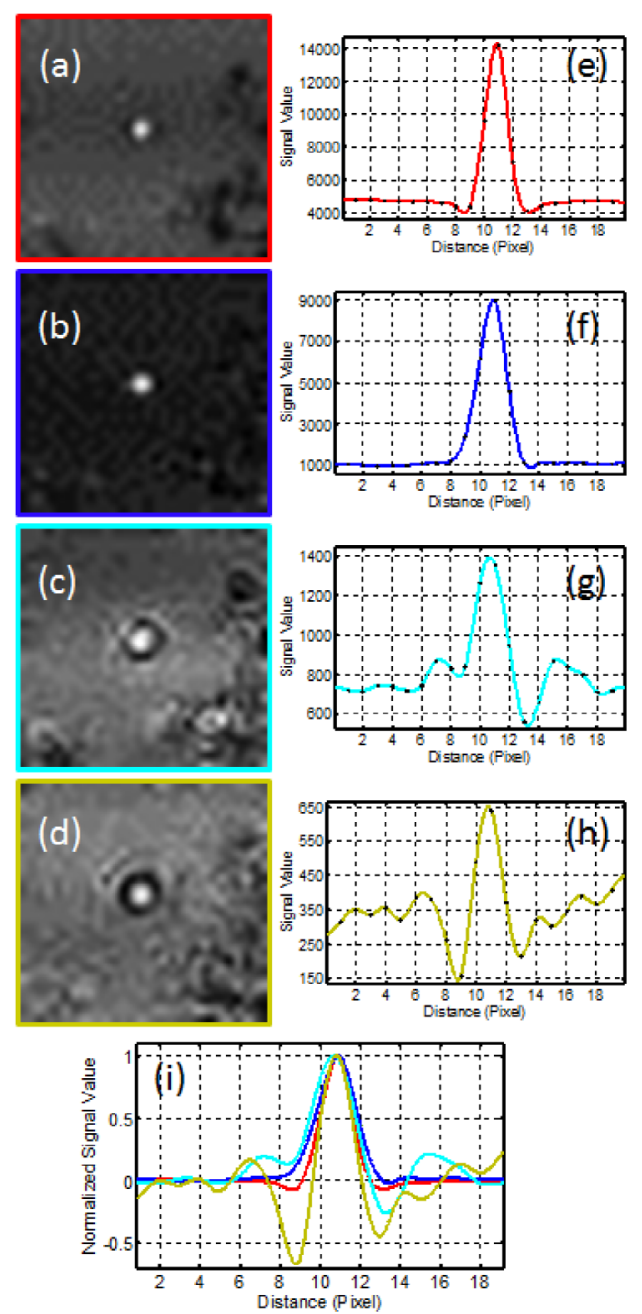

Fig. 3. Gold nanoparticle images for various levels of defocus using the FFOCT setup of Figure 1. FFOCT images $(\mathrm{a}-\mathrm{d})$ and the corresponding intensity profile $(\mathrm{e}-\mathrm{h})$ of a targeted nanoparticle are shown for well-focused (a,e) and defoused for $10 \mathrm{um}(\mathrm{b}, \mathrm{f}), 20 \mathrm{um}(\mathrm{c}, \mathrm{g})$ and $30 \mathrm{um}(\mathrm{d}, \mathrm{h})$ situations. Normalized PSF profiles are shown in (i) indicating no obvious broadening are observed after inducing different level of defocus [14].

or intensity convolution of the diffraction-limited images with the aberrated PSF. Depending on the nature of the illumination, spatially coherent or incoherent, amplitude or intensity has to be considered $[15,16]$. Here, we will pay attention to the system PSF of interferometric imaging systems for which an undistorted wavefront from a reference beam interferes with the distorted wavefront of the object beam.

To express the unique behavior of FFOCT that uses spatially incoherent sources we will successively consider the cases of scanning OCT with spatially coherent illumination, wide-field OCT with spatially coherent illumination and FFOCT with spatially incoherent illumination to show that in FFOCT with incoherent illumination the system PSF width is almost independent of the aberrations and that only its amplitude varies. 

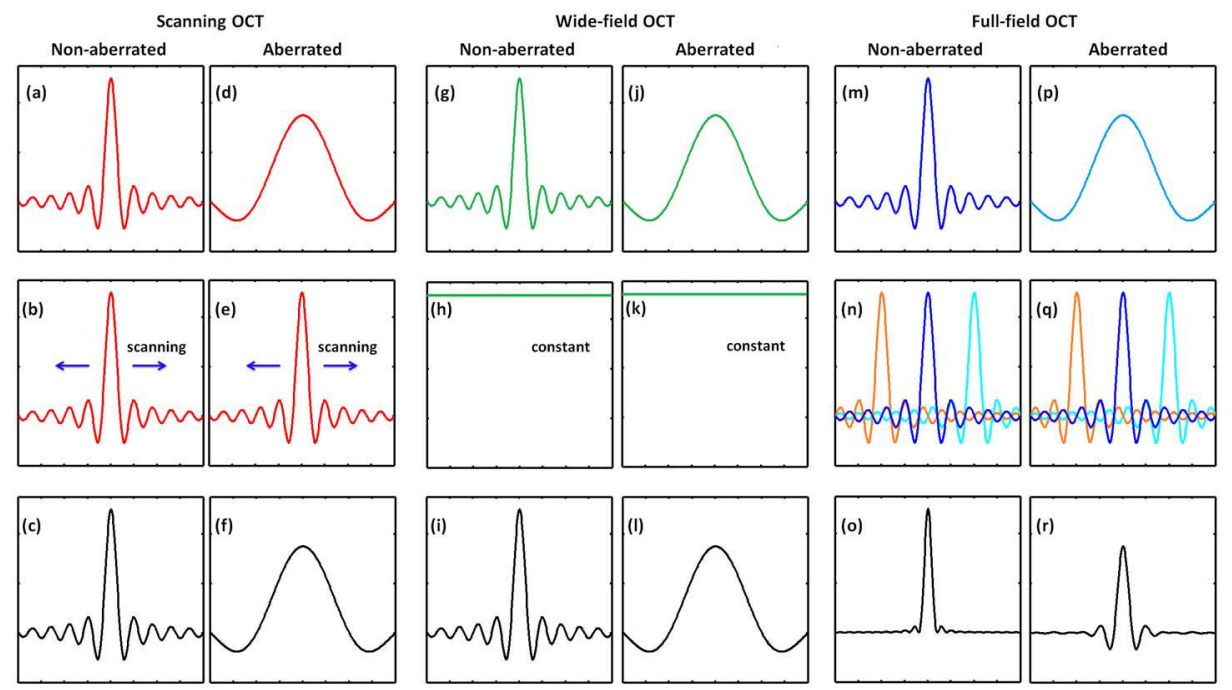

Fig. 4. Illustration of single point scatterer (PSF) interferences in both non-aberrated and aberrated sample arm PSF situations for scanning OCT and wide-field OCT with spatially coherent illumination and FFOCT with spatially incoherent illumination. (a,g,m) Non-aberrated sample arm PSF, (d,j,p) Aberrated sample arm PSF, (b,e) Scanning reference arm PSF for scanning OCT, $(\mathrm{h}, \mathrm{k})$ Constant reference field for wide-field OCT, $(\mathrm{n}, \mathrm{q})$ Reference arm PSFs for FFOCT, $(c, f, i, l, o, r)$ The corresponding interference signal (system $\mathrm{PSF})$. Different colors in $(\mathrm{n}, \mathrm{q})$ indicate the spatial incoherence from each other [14].

\subsection{From scanning OCT to coherent and incoherent FFOCT: basic calculations}

We will consider a point scatterer as our object and will analyze the system response to such object in order to stick to the PSF definition, this scatterer is located at $\left(x^{\prime}, y^{\prime}\right)=$ $(a, b)$, we call the sample arm PSF of the interferometer $h_{s}$ and the diffraction limited reference arm PSF of the interferometer $h_{r}$. We will ignore all the constant factors in the following expressions. So in all the three cases, the sample field at the detection plane would be

$$
g_{s}=h_{s}\left(x^{\prime}-a, y^{\prime}-b\right)
$$

In the case of traditional scanning OCT, the reference field of each scanning position at the detection plane would be $h_{r}\left(x-x^{\prime}, y-y^{\prime}\right)$. The final interference would be a sum of the interference term across the scanned field:

$$
\left\langle g_{s} g_{r}\right\rangle_{s}=\iint h_{s}\left(x^{\prime}-a, y^{\prime}-b\right) h_{r}\left(x-x^{\prime}, y-y^{\prime}\right) d x^{\prime} d y^{\prime} .
$$

Thus, the PSF of scanning OCT system would be a convolution of the sample arm PSF and the reference arm PSF as shown in Figures $4 \mathrm{a}-4 \mathrm{c}$. When aberrations exist, the convolution of the aberrated sample arm PSF with the diffraction-limited reference arm PSF results in an aberrated system PSF for the scanning OCT systems (Figs. 4d-4f).

In the case of wide-field OCT, coherent sources are typically expanded by lenses to form parallel illuminations on both arms of the interferometer [10]. Thus plane waves impinge on both the object and the reference mirror. In the sample arm, the point scatterer will send a spherical wave back. On the camera plane, the beam will be focused and can be described by expression (1). For the reference arm with a 


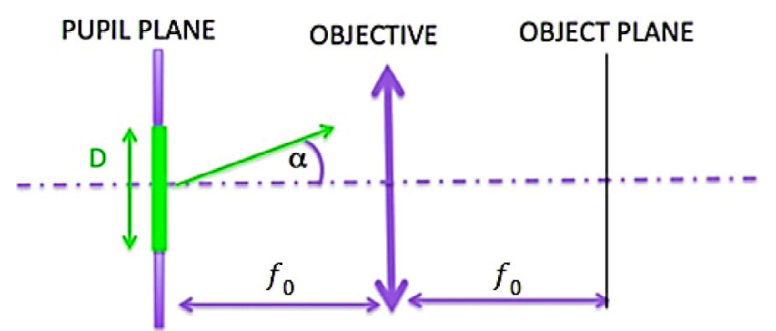

Fig. 5. Illustration of the Van Cittert-Zernike theorem defining the coherence angle $\alpha$ in the case of an objective with a focal length $f_{0}$ and pupil diameter $D$.

reflective mirror, consider homogeneous illumination is achieved, a plane wave will be reflected back and form a uniform field at the camera plane. Thus the interference between the two arms would be

$$
\left\langle g_{s} g_{r}\right\rangle_{w}=h_{s}\left(x^{\prime}-a, y^{\prime}-b\right) .
$$

So the system PSF is actually defined by the sample PSF. It is illustrated in Figures $4 \mathrm{~g}-4 \mathrm{i}$. When aberrations distort the backscattered wavefront of the sample arm, the aberrated sample arm PSF interferes with a uniform reference field results in an aberrated system PSF for the wide-filed OCT systems (Figs. 4j-4l).

In the case of FFOCT with spatially incoherent illumination, we have to consider the definition of the spatial coherence of the beams that impinge the reference arm as well as the sample arm of the interferometer. As shown in Figure 5, let's consider a circular uniform incoherent source located in the image focal plane of a microscope objective with a focal length of $f_{0}$, as in a Koehler illumination setup.

The first step is to determine the extension of the spatial coherence area in the field of view. Here we have used a fully spatially incoherent source: the spatial coherence limitation is thus inked to the numerical aperture of the optics behind the source. In this case the Van Cittert-Zernike theorem states that the coherence angle is given by the Fourier transform of the source luminance [17]. With a pupil diameter of $D$, the angle would be defined as $\sin \alpha=1.22 \lambda / D$. At the level of focal plane, it corresponds to a zone of radius $\rho=f_{0} \lambda / D$ or $\rho=1.22 \lambda / 2 N A$. We can say that the focal plane is "paved" by small coherent areas $(\mathrm{CA})$ of radius $\rho$ when there is no aberration. In fact, the $\mathrm{CA}$ radius is also the radius of the diffraction spot that limits the resolution of the microscope objective in absence of aberrations. When going from one diffraction spot to the next adjacent diffraction spots the incoherent plane waves impinging the objective are separated by $\pm \lambda$ on the edges of the pupil.

In absence of aberrations for a FFOCT system, the single point scatterer at the object plane of the sample arm lies in a single CA (Fig. 6a) and the backscattered signal will only interfere with signal reflected from the corresponding $\mathrm{CA}$ in the reference arm (Fig. 6c). Note that the size of the CAs is the same as the diffraction spot, the signal from one CA at the camera plane could be expressed as the reference PSF. Thus the interference would be

$$
\left\langle g_{s} g_{r}\right\rangle_{f}=h_{s}\left(x^{\prime}-a, y^{\prime}-b\right) h_{r}\left(x^{\prime}-a, y^{\prime}-b\right) .
$$

The system PSF is actually the dot product of the sample PSF and the reference corresponding coherent PSF as shown in Figures 4m-4o. The overall signal reflected from the reference mirror at the camera is still homogenous but we displayed it by combining multiple reference PSFs reflected from different CAs that have different spatial modes and do not interfere with the object PSF. 


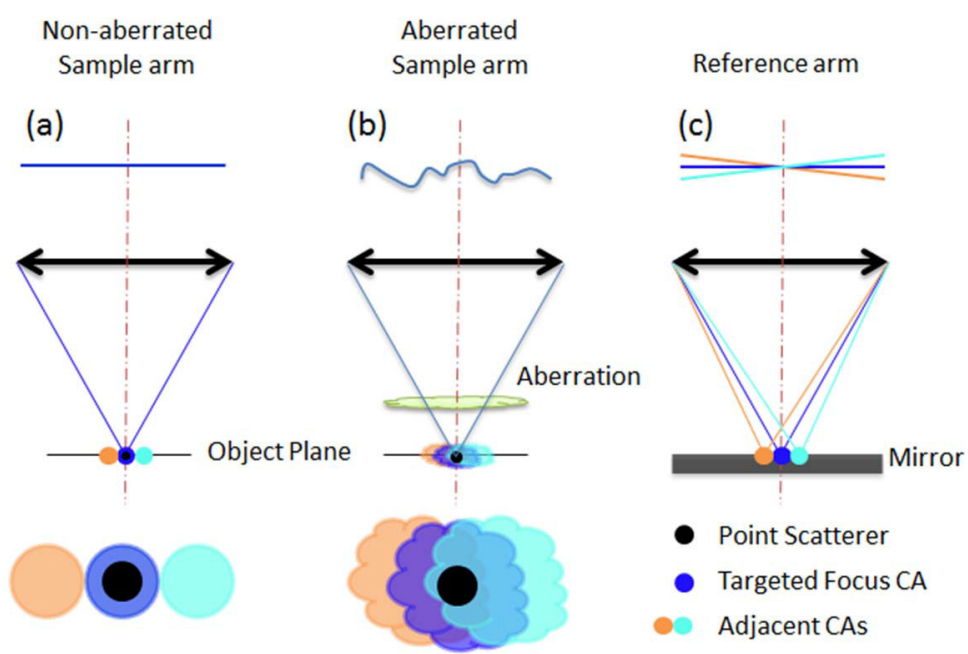

Fig. 6. Illustration of the sample and reference wavefronts in spatially incoherent interferometer with a single point scatterer in cases of non-aberrated and aberrated sample arm. Different colors in CAs and wavefronts indicate different spatial modes [14].

When aberrations distort the wavefront in the sample arm, the various CAs in the object plane will be distorted, have larger sizes and overlap with each other (Fig. 6b). This results in the backscattered signal of the single point scatterer in the sample arm containing not only the spatial mode of the targeted focus CA but also the modes from the overlapped adjacent CAs. Thus with aberrations that create a broadened sample PSF, interference will happen not only with the reference beam corresponding to the targeted $\mathrm{CA}$, but also with the beams corresponding to the adjacent CAs.

What we are going to demonstrate and to illustrate is that the interference signal with the targeted focus CA gives a much stronger signal than the one with the adjacent CAs resulting in a final PSF that is much thinner than the one of the broadened sample PSF. At the level of the image plane, the interference between the sample aberrated beam and the non aberrated reference beam is only possible in a zone limited by the spatial coherence of the reference beam. In order to be more quantitative, we will use the Strehl ratio approach. When aberrations are present astronomers are used to introduce the "best focus" signal intensity damping compared to the diffractionlimited conditions that is given (for small aberrations) by the Strehl ratio $S=e^{-\sigma^{2}}$. $S$ is proportionnal to the peak aberrated image intensity; $\sigma$ is the root mean square deviation over the aperture of the wavefront phase $\sigma^{2}=(\operatorname{std}(\phi))^{2}$. Suppose $\phi$ is the phase of the interference wavefront between the sample signal and the reference signal corresponding to the targeted focus $\mathrm{CA}$, then the phase of the interference wavefront with the reference signals corresponding to an adjacent CAs is $\phi+\phi_{1}$, where $\phi_{1}$ is a phase that varies linearly from one edge of the pupil to the other in the range of $\pm 2 \pi$. A comparison between the signal ratio of the interference (amplitude) signal with the targeted $\mathrm{CA}$ and the one with an adjacent $\mathrm{CAs}$ is now:

$$
s_{t}=e^{-(s t d(\phi))^{2}} \gg s_{a}=e^{-\left(s t d\left(\phi+\phi_{1}\right)\right)^{2}} .
$$

This new phase shift acts as a supplementary aberration and thus would show off axis CAs signals are more strongly damped than the targeted CA.

Indeed we can consider various aberrations leading to a Strehl ratio of 0.3 , numerical calculations results are shown in Figure 7. For defocus, the intensity 


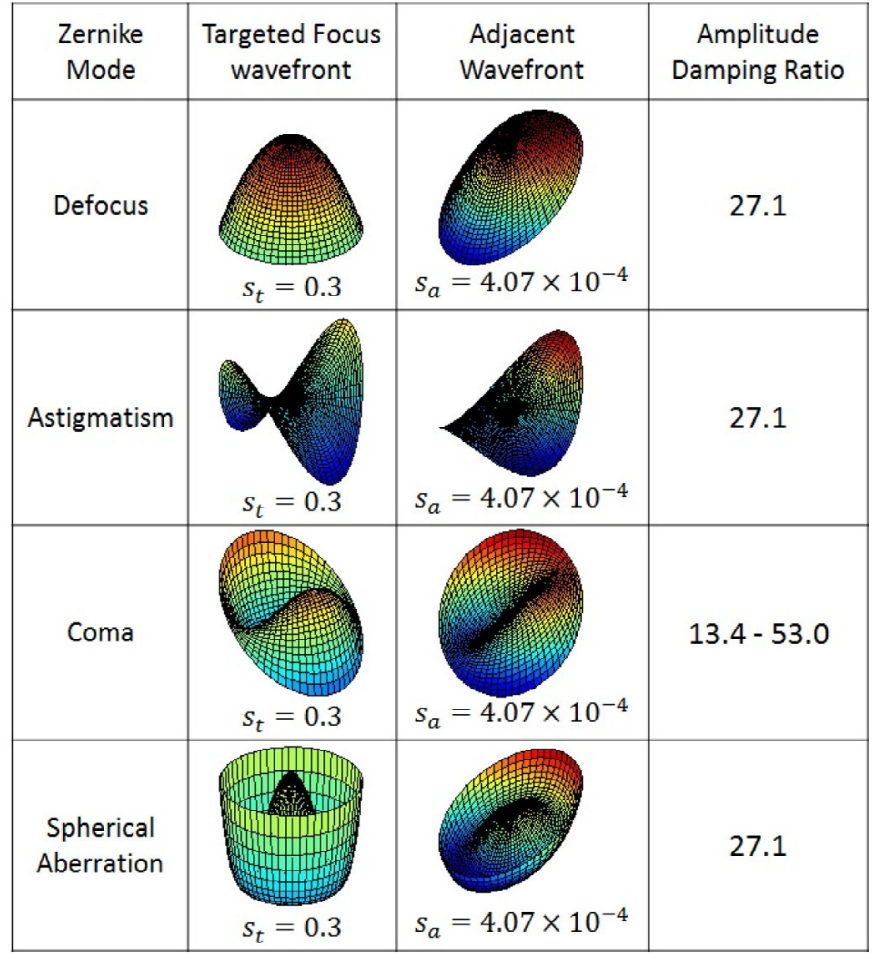

Fig. 7. Aberrated interference wavefronts and numerical simulations of the Strehl ratio and amplitude damping for interference with targeted CA and adjacent CAs. Defocus, astigmatism, coma and spherical aberration are considered. The damping for coma varies depending on the spatial position of the adjacent CAs [14].

ratio of the interference with adjacent CAs is damped for about 740 times compared with the interference with the targeted focus CA, resulting in a signal damping or an amplitude damping of 27.1 times. The amplitude damping ratio is then $\sqrt{s_{t} / s_{a}}$ as amplitude instead of intensity is obtained in FFOCT signal. It's easy to prove that this value is fixed for all the axisymmetric aberrations like defocus, astigmatism, spherical aberrations, etc. While for coma with a Strehl ratio of 0.3, the simulated amplitude damping ratio is $13.4-53.0$ times depending on the spatial position of the adjacent CAs. In another word, the interference signal was severely damped going from the targeted CA to the adjacent CAs. Thus in the camera plane, as shown in Figures $4 \mathrm{p}-4 \mathrm{r}$, the interference signal results in a dot product of the aberrated sample PSF with the reference PSF corresponding to the targeted focus CA since the interference with the reference PSFs corresponding to the adjacent CAs are significantly reduced. This actually matches with equation (4) for non-aberrated situation, the system PSF could be calculated by the dot product of the sample PSF and the reference PSF. For distorted sample PSF (mostly broadened), its interference with the reference channel conserves the width of an unperturbed PSF with only a reduction in the FFOCT signal level. The shape of the PSF in presence of aberration looks more and more as the amplitude PSF with larger wings. We mentioned "almost" for the resolution conservation, because there are situations in which the product of the reference arm PSF with off-center aberrated sample arm PSF may results in losing some sharpness due to the high side lobes of the amplitude Bessel PSF function that are larger than for its square when dealing with intensities. 


\section{Simplifying adaptive optics for low order aberrations}

The ultimate goal of our study is to apply FFOCT for human eye examinations. In retinal examinations, despite of scattering problems inside the retinal tissues, there are also multi-scale aberrating structures in the eye that could degrade the optical images quality. Thus a wavefront adaptive system is typically needed to achieve diffraction-limited imaging. Originally proposed and developed for astronomical optical telescopes to correct the atmosphere-induced aberrations [18,19], adaptive optics (AO) has found valuable applications to correct biological tissue-induced aberrations in biological and medical imaging [20], especially for retinal imaging to visualize cellular structures [21-23]. AO assisted fundus photography [24], scanning laser ophthalmoscopy [25-27] and OCT [28-35] systems have achieved reliable images of cones and rods photoreceptors.

In many systems such as two-photon microscopy [36] and AO-OCT systems $[28,29,31-35,37]$, the AO part usually uses a conjugation of the image focal plane of the microscope objective or of the eye pupil with the wavefront sensors or correction devices. Since one cannot rely on simple geometrical optics propagation of the wavefront but one has to account for diffractive effects of wave propagation for high order aberrations, this strict pupil conjugation appears to be mandatory when very high order aberrations are involved. However, the telescopic systems for strict pupil conjugation would increase the system complexity and the optical path length, which would be difficult for FFOCT system since the two arms have to be balanced within less than one micrometer due to the axial sectioning of FFOCT.

This would be different for low order aberrations. Many studies on eye aberrations have shown that the majority of the Zernike polynomials that are involved in a large number of eyes aberrations tests are mostly low order ones [38-40], meaning that the wavefront would barely change at different steps of the propagation. So in order to simplify the setups and to be able to apply AO-FFOCT to low order aberration corrections, a transmissive wavefront corrector that could be roughly set in the beam path without strict conjugation would be enough, analogous to commonly used spectacles for correcting eye's myopia and astigmatism. Transmissive liquid crystal spatial light modulator (LCSLM) [41-45] fits well for our application as it can work in transmissive way with a large number of pixels and a low control voltage. LCSLMs have already been used to alter the refractive state [44] or to correct the aberrations of the eye [45]. The confined $2 \pi$ phase-modulation range of LCSLM might limit the correction of aberrations with large magnitudes. But the adjusting range is doubled as the incoming and outgoing beams both induce optical path difference in our system. Whatever is the correcting system, reflective or transmissive pupil conjugation have been used in both cases as this is done in astronomy for a small field of view. Finally either phase wrapping could be used to extend the dynamic range $[44,46]$ or simple visual corrections for defocus and astigmatism could be added to the sample optical path.

In most $\mathrm{AO}$ systems, direct wavefront measurements are usually conducted with a wavefront sensor or coherence-gated wavefront sensing in a closed-loop configuration together with a wavefront corrector. Due to the lack or generally usable wavefront sensors and the inherent complexity of the coherent wavefront sensing, considerable interests have been focused on wavefront sensorless methods such as hill climbing [47], genetic algorithm [48], simulated annealing [49], pupil segmentation [50], etc. As discussed before, aberrations do not affect the width of the PSF but only the signal level in FFOCT. Therefore, a wavefront sensorless method that relies on the improvement of image signal or signal-to-noise ratio looks naturally well adapted to the FFOCT detection [51-54] and was used for the optimization process that simplified the AO-FFOCT system. 


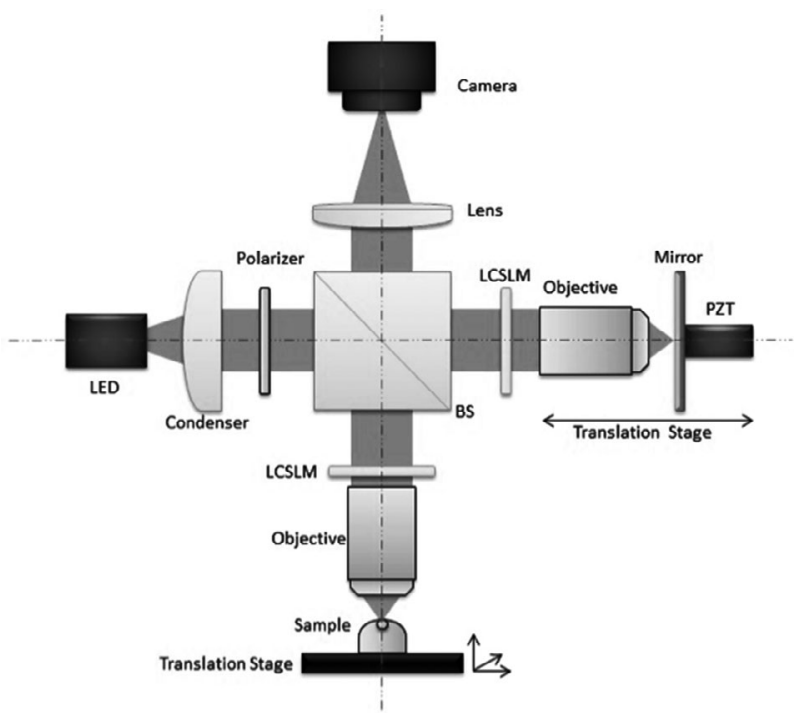

Fig. 8. Schematic of AO-FFOCT system coupled with LCSLMs. BS: beamsplitter, LCSLM: liquid crystal spatial light modulator, PZT: piezoelectric transducer [55].

\section{A compact AO-FFOCT setup}

The current AO-FFOCT system schematic is shown in Figure 8. The main part is the typical FFOCT system based on a Linnik interferometer. A LED (660 nm center wavelength, $25 \mathrm{~nm}$ bandwidth, Thorlabs) is used as the incoherent light source. The illumination beam is split into the reference arm and the sample arm at a ratio of 50:50 with a non-polarizing beamsplitter. Two Nikon 4X/0.2NA Plan APO objectives are used, one is in the sample arm to simulate the open pupil human eye and the other is in the reference arm. A reference mirror supported by a piezoelectric transducer is placed at the focal plan of the objective in the reference arm while the imaging object is placed in the sample arm. The back-reflected beams from the reference mirror and the sample are recombined by the beamsplitter and imaged with an achromatic doublet lens onto a fast (150 fps) CMOS camera (MV-D1024E-160-CL-12, PhotonFocus). The setup is aligned to ensure that the focusing of the two arms and their optical paths are matched. The piezoelectric transducer creates a four-phase modulation of the reference arm and a FFOCT image can be reconstructed with these four corresponding images [12]. Usually several FFOCT images are averaged for improving the signal to noise ratio (SNR). 5 images were used for the following described experiments requiring about $150 \mathrm{~ms}$. The system has a field of view of $1.7 \times 1.7 \mathrm{~mm}^{2}$, and the theoretical resolutions are $2 \mu \mathrm{m}$ (transverse) and $7.7 \mu \mathrm{m}$ (axial).

For conducting the wavefront correction, a transmissive LCSLM is installed in the sample arm at about $2.5 \mathrm{~cm}$ after the back aperture of the objective lens, while another identical LCSLM is set in the reference arm for dispersion correction. A polarizer is inserted in the illumination path since the LCSLM works only with polarized light. By electronically varying the orientation of the molecules inside the pixels of the LCSLM, the refractive index of the pixels is changed independently from each other, resulting in variable retardance abilities to the polarized light passing through them. 


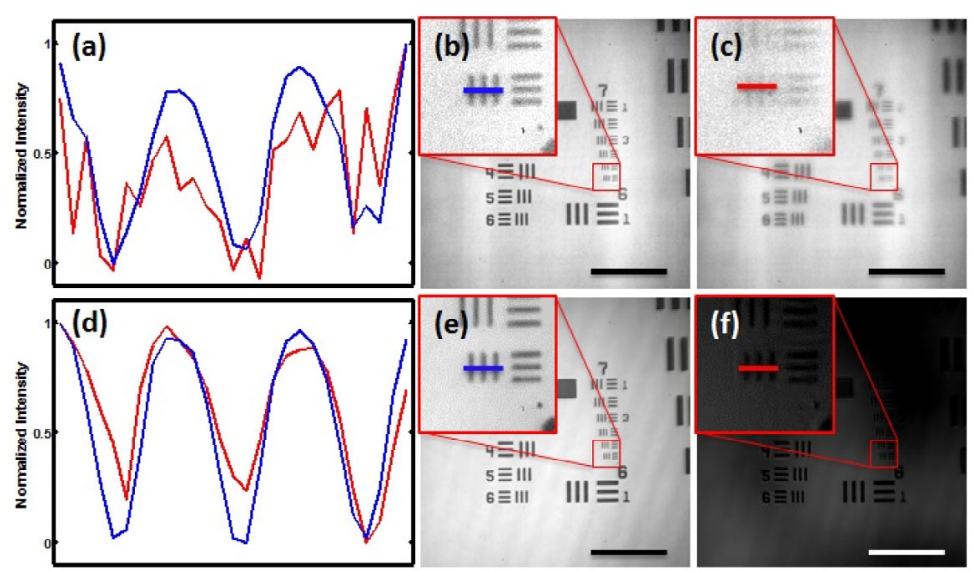

Fig. 9. Comparison of the reflectance $(b, c)$ and FFOCT (e,f) images of a negative USAF resolution target before $(\mathrm{b}, \mathrm{e})$ and after $(\mathrm{c}, \mathrm{f})$ adding a random aberration; $(\mathrm{a}, \mathrm{d})$ show the comparison of the normalized reflectance intensity and FFOCT signal of the selected line without (blue) and with (red) aberration added. Scale bar: $100 \mu \mathrm{m}$ [55].

\subsection{Free space aberration: USAF resolution target without blurring}

Using a negative USAF setting at the best focus position of the sample arm of the customized AO-FFOCT system, the resolution conservation merit of FFOCT system was again confirmed experimentally. Here, a random aberration $($ Strehl ratio $=0.06)$ was induced with the LCSLM in the sample arm by generating and applying random voltages within the adjusting range across the LCSLM pixels. Figure 9 shows the sample reflectance images and FFOCT images of the USAF resolution target before and after the random aberration was induced. The reflectance images were recorded by blocking the reference arm in FFOCT thus the system works as a wide-field microscope. The reflectance image is blurred after the aberration is added, while there is no obvious blurring of the line patterns in the FFOCT image and only a reduction of the image intensity could be noticed. The normalized intensity of the selected line in the reflectance image shows a distortion after the aberration was added, while it shows a conservation of the shape for the FFOCT image. Note that the image contrast of scanning OCT using spatially coherent illumination would be close to the reflectance image from the sample arm.

\subsection{Aberration correction algorithm}

Since aberrations affect only the signal level without reducing the image resolution in FFOCT, a wavefront sensorless approach based on the FFOCT signal level is used for aberration correction. This method consists of the sequential adjustment of the coefficients of low order orthogonal Zernike polynomial functions applied to the LCSLM to optimize the metric function. The mean intensity of FFOCT image was used as the metric function for LCSLM-induced aberration correction with USAF resolution target as the sample. For in-depth sample-induced aberration correction, the average intensity of the 300 pixels with maximum intensity values in the FFOCT image was used as the metric function. This is due to the mean intensity of the overall image would be less sensitive to the AO process since most parts of the FFOCT image has very low or even no signal. Of course the optimization process could also be restricted 
to specific region of interest. Indeed anisoplanatism does exist as demonstrated in Figure 11, but the experiment results show acceptable correction with this simple AO algorithm. No phase wrapping was used for experiments here because the magnitude of the wavefront distortions to be compensated was within the dynamical range of the SLM. Coefficients were indeed selected within the adjusting range of the LCSLM. The orthogonality of different Zernike modes ensures that the coefficient of each mode for optimal correction is determined independently $[56,57]$. This algorithm has been proposed and used by many groups with different wavefront shaping methods and optimization metrics in specific applications $[35,58,59]$. For the aberration correction experiments mentioned here, only Zernike modes 3 to 8 were optimized just to demonstrate the feasibility of our system and method. For each mode, FFOCT images were taken for 7 different coefficients within the adjusting range. With the extracted metric function values, B-spline interpolations were done and the coefficient that produced the highest metric function was chosen as the correction value.

\section{LCSLM-induced aberration correction}

Experiments of LCSLM-induced aberration correction were first conducted by imaging a negative USAF resolution target in order to validate the performances of the non-conjugate AO-FFOCT system with wavefront sensorless algorithm. As shown in Figure 10, in this experiment LCSLM2 were inserted into the sample arm for aberration introduction at about $5 \mathrm{~cm}$ after the original LCSLM1 which was used for aberration correction, thus there is no well-defined conjugation between the aberration introduction plane and the correction plane. A glass slide was inserted into the reference arm for dispersion compensation. The USAF target was set at the best focus position in the sample arm and a random aberration mask (Strehl ratio $=0.12$ ) was generated and applied to the LCSLM2. Figure 11a shows the original FFOCT image with the added aberration. By using the wavefront correction algorithm and applying the correction phase mask onto LCSLM1, defocus, astigmatism, coma and spherical aberration were corrected sequentially. Figures $11 \mathrm{~b}-11 \mathrm{~g}$ show the images after each correction with a clearly visible improvement of image quality after each optimization process. The black curve in Figure 11h shows the increase of the metric function and the red, blue and green dashed curves display the mean intensity changes of the corresponding selected regions indicated with the same colors in Figures 11a and 11g. The fact that different levels of improvement were achieved for different regions with the same correction phase mask for each Zernike mode implies the existence of anisoplanatism in our experiment. Nevertheless, the mean intensity of the FFOCT image got an increase of $135 \%$ after the overall correction, reaching $80 \%$ of the non-aberrated FFOCT image, while having diffraction-limited resolution. The Strehl ratio was increased by a factor of 5.3 to a value of 0.64 .

For comparison, conjugate AO experiment by using the same LCSLM for aberration introduction and correction was conducted. With the same random aberration induced by LCSLM2, aberration correction was demonstrated also on LCSLM2. With the same algorithm, Zernike modes $3-8$ was corrected by applying net voltages of random pattern plus the Zernike modes to LCSLM2. As shown in Figure 12, the whole correction result in the mean intensity of the FFOCT image reaching $86 \%$ of the non-aberrated FFOCT image. The Strehl ratio was increased by a factor of 6.2 to a value of 0.74 . 


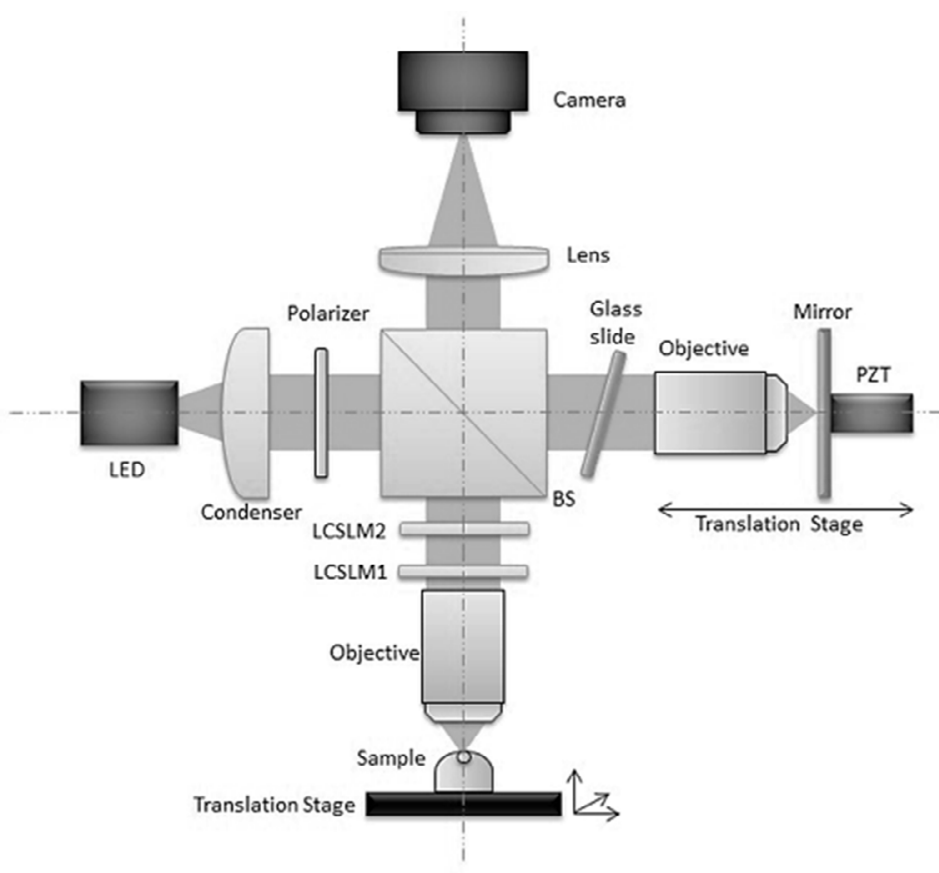

Fig. 10. Schematic of adaptive optics FFOCT system for LCSLM-induced aberration correction. LCSLM2 was inserted at $50 \mathrm{~mm}$ after LCSLM1. LCSLM2 was used for aberration introduction while LCSLM1 was used for aberration correction [55].

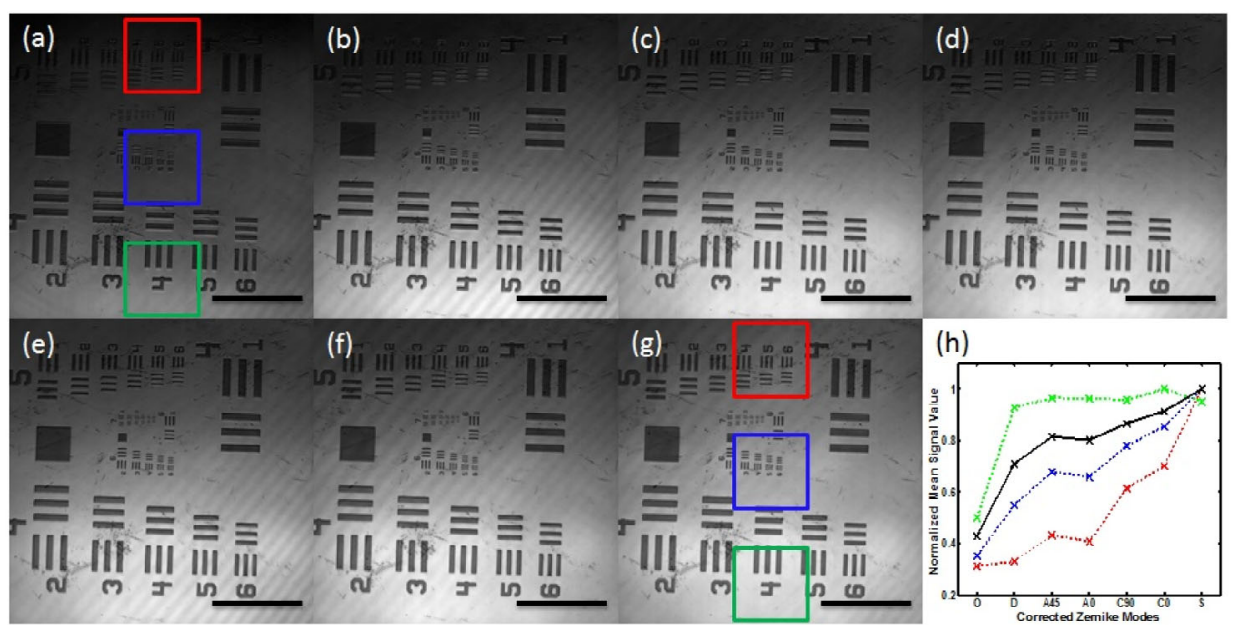

Fig. 11. FFOCT images of a negative USAF resolution target during the non-conjugate $\mathrm{AO}$ correction process of a random aberration. (a) Original image with a random aberration added, (b-g) images after defocus, astigmatism 45, astigmatism 0, coma 90, coma 0 and spherical aberration were corrected respectively, (h) graph of the metric function (black curve) increase after each correction step and mean intensity changes (red, blue and green dashed curves) of the corresponding selected regions indicated in $(\mathrm{a}, \mathrm{g})$. Scale bar: $350 \mu \mathrm{m}[55]$. 

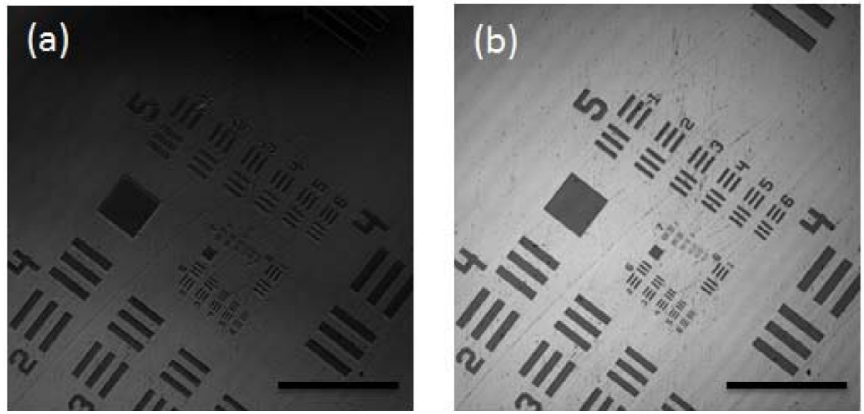

Fig. 12. FFOCT images of a negative USAF resolution target before and after the conjugate $\mathrm{AO}$ correction process of a random aberration. (a) Original image with a random aberration added, (b) image after defocus, astigmatism 45, astigmatism 0 , coma 90 , coma 0 and spherical aberration were corrected. Scale bar: $350 \mu \mathrm{m}$ [55].

\section{Sample induced aberrations correction}

\subsection{Ficus leaf experiment: weak aberrations correction}

Due to the spatial variations of refractive index within biological samples and surface topography, aberration distortion is severe when imaging into the sample volume. In order to further demonstrate the feasibility of our system and method even for weak aberrations correction, experiments of sample induced aberrations corrections were done with a ficus leaf. The system setup described in Figure 3 was used here. By imaging at a depth of $75 \mu \mathrm{m}$ under the leaf surface only weak aberrations are induced and we can thus check the sensitivity of our correction approach; the low order contents of the self-induced sample aberrations were corrected step by step with the aforementioned methods. As shown in Figure 13, the optimized image (Fig. 13b) shows an intensity increase compared with the original image (Fig. 13a) and from the zoomed in images, more structured information appears. This is due to the fact that the correction process increased the SNR and more signals that were buried by the noise before appear after the AO correction. The graph of the metric function while adjusting the coefficients of each Zernike mode is displayed in Figure 13c. The highest positions of each curve correspond to the coefficients used for the optimal correction of each mode. Figure 13d shows the increase of metric function. The whole correction process results in $13.3 \%$ improvement of the metric function. The metric function improvement increases to $35.5 \%$ when imaging deeper at $120 \mu \mathrm{m}$ under the leaf surface in another experiment.

\subsection{Mouse brain slice: strong aberrations correction}

After showing the ability of this AO-FFOCT approach to optimize the signal even with a low level of aberration, we checked another biological tissue of relevance that suffers from strong scattering and stronger aberrations - the brain tissue, where FFOCT signal is usually strongly reduced when imaging deep in the sample. Experiments were conducted with a fixed mouse brain tissue slice to correct the wavefront distortion. Imaging was performed at $50 \mu \mathrm{m}$ under the brain tissue surface without liquid matching fluid and the results are shown in Figure 14. The high-signal fiberlike myelin structures appeared much more clearly after the whole correction process because of the increased SNR; indeed the metric function was increased by $121 \%$. 
(a)
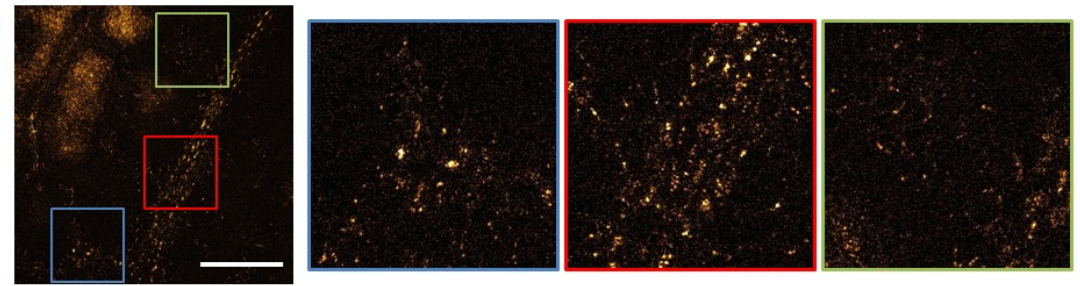

(b)
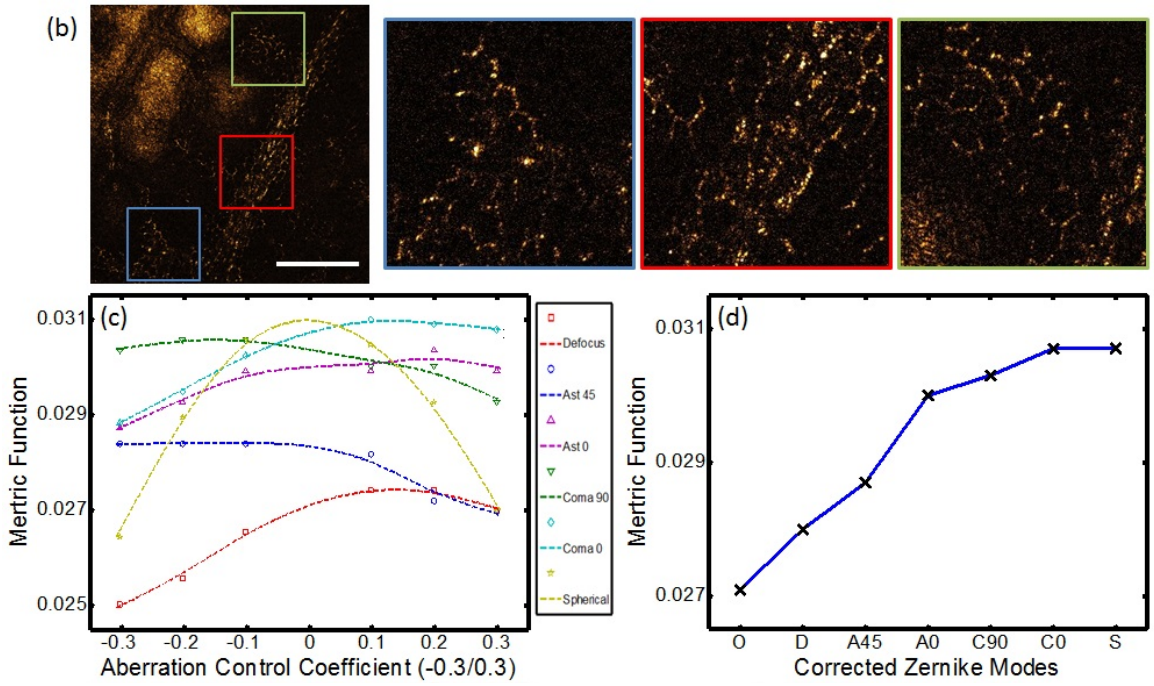

Fig. 13. Comparison of FFOCT images of a ficus leaf before (a) and after (b) sample selfinduced aberration was corrected when imaging at a depth of $75 \mu \mathrm{m}$. (c) Graph of the metric function during the optimization process, (d) graph of the metric function increase after each correction step. Scale bar: $500 \mu \mathrm{m}$, Zoomed in area: $425 \times 425 \mu \mathrm{m}$ [55].
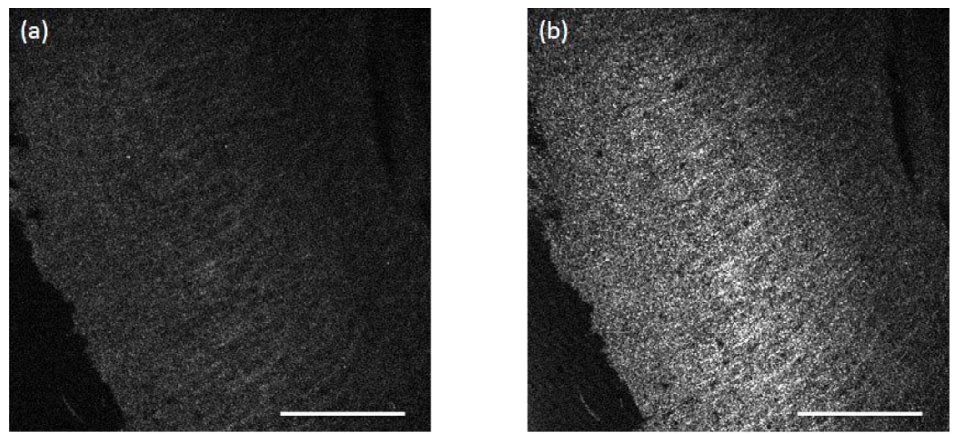

Fig. 14. Comparison of FFOCT images of fixed mouse brain tissue slice before (a) and after (b) sample self-induced aberration was corrected when imaging at a depth of $50 \mu \mathrm{m}$. Scale bar: $500 \mu \mathrm{m}[55]$.

\section{Discussion and conclusion}

We have shown that in spatially incoherent illumination interferometry like full-field OCT, the system PSF width is almost insensitive to aberrations with only signal amplitude reduction. This is demonstrated by a simple theoretical analysis as well as numerical simulations for different aberrations, and confirmed by experiments with 

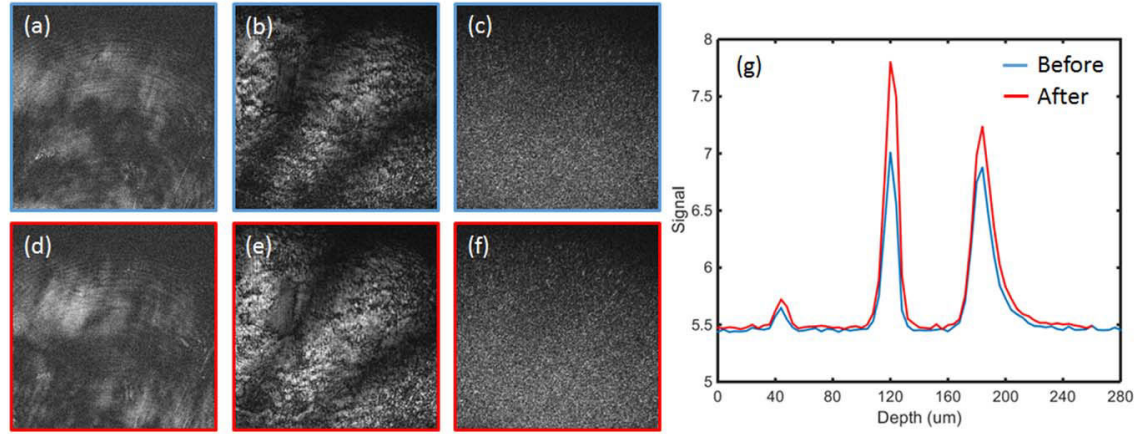

Fig. 15. AO-FFOCT images of different retinal layers of an artificial eye before $(\mathrm{a}-\mathrm{c})$ and after $(\mathrm{d}-\mathrm{f})$ aberration correction and averaged image intensity curves along depth scanning (g) showing the signal increase of all the three detected layers.

a full-field OCT system. More precisely the aberration-induced reduction in signal is roughly proportional to the square root of the Strehl ratio. Let us consider the realistic case of a diffraction-limited imaging system with a PSF width of $2 \mu \mathrm{m}$ that allows for instance resolving the cones in retinal imaging. With a Strehl ratio of 0.1, which is considered to give a low quality image, the PSF would be broadened to about $6 \mu \mathrm{m}$ that would mask the cell structures. But in full-field OCT system, the same Strehl ratio would only reduce the signal by a factor of 3.1 while keeping the image sharpness.

We also demonstrated that a compact transmissive LCSLM can be directly coupled to an FFOCT system as an AO element for wavefront distortion compensation with a wavefront sensorless algorithm. Our experiments show the potential of this compact AO-FFOCT system for aberrations correction imaging. The conjugation of the LCSLM with the pupil plane was discarded in our AO-FFOCT system. Traditionally, AO devices are usually conjugated with a well-defined plane. For both pupil $\mathrm{AO}$, in which conjugation is done to the pupil plane, and conjugate $\mathrm{AO}$, in which conjugation is done to the plane where the aberrations dominate, a plane is needed for wavefront measurement and the inverse phase mask needs to be applied to the same plane with the conjugated wavefront correctors. The advantages and disadvantages of both conjugations have been recently discussed in [60]. From what we have learned in our experiments, we think that the problem might be easier for applications with metric-based wavefront sensorless adaptive optics because the only criteria are the metric functions of the image. Strict conjugation might be abandoned, especially for low order aberrations correction cases. The corrected signal level with this non-conjugate $\mathrm{AO}$ reaches $80 \%$ of the non-aberrated situation. This is slightly inferior but still acceptable compared with a conjugate $\mathrm{AO}$ experiment which results in a corrected FFOCT image signal level reaching $86 \%$ of the non-aberrated image.

Our approach simulating eye aberration correction in a simple manner opens the path to a straightforward implementation of AO-FFOCT for retinal examinations in the future research. In the case of eye examinations, we can restrict aberrations correction to the main aberrations (e.g., focus and astigmatism) that will improve the SNR and skip the high order aberrations. Ultimately, the lens in the eyeball will play the role of the objective used in the sample arm in our experiments, therefore a new reference arm with path and dispersion compensation [61] will need to be designed taking into consideration of the eye characteristics. By using an artificial eye model used to train ophthalmologists [62], we have demonstrated preliminary adaptive optics retinal imaging experiments. By translating the model eye along the 
optical axis, three retina layers were detected. The corresponding FFOCT images are displayed in Figures 15a-15c. Based on the second layer, the model eye self-induced aberrations corrections were done to improve the image signal level by using the same algorithm as described before. After the optimization process, the improved FFOCT retinal images are shown in Figures $15 \mathrm{~d}-15 \mathrm{f}$. The curves in Figure $15 \mathrm{~g}$ shows the average image intensity of the FFOCT images along different depth while the peaks indicating the three detected layers. The signal level for all the layers is increased after aberration correction. Taking the second layer as an example, the signal level has increased by $48 \%$ after subtracting the background noise.

This work is supported by the HELMOLTZ Synergy funded by the European Research Council (ERC).

\section{References}

1. T. Wilson, in Confocal Microscopy (Academic Press: London, 1990), Vol. 426, pp. 1-64

2. W. Denk, J.H. Strickler, W.W. Webb, Two-photon laser scanning fluorescence microscopy, Science 248, 73 (1990)

3. D. Huang, E.A. Swanson, C.P. Lin, J.S. Schuman, W.G. Stinson, W. Chang, M.R. Hee, T. Flotte, K. Gregory, C.A. Puliafito, J.G. Fujimoto, Optical coherence tomography, Science 254, 1178 (1991)

4. N. Nassif, B. Cense, B.H. Park, S.H. Yun, T.C. Chen, B.E. Bouma, G.J. Tearney, J.F. de Boer, In vivo human retinal imaging by ultrahigh-speed spectral domain optical coherence tomography, Opt. Lett. 29, 480 (2004)

5. R. Huber, M. Wojtkowski, J.G. Fujimoto, Fourier Domain Mode Locking (FDML): A new laser operating regime and applications for optical coherence tomography, Opt. Express 14, 3225 (2006)

6. B.M. Hoeling, A.D. Fernandez, R.C. Haskell, E. Huang, W.R. Myers, D.C. Petersen, S.E. Ungersma, R.Wang, M.E. Williams, S.E. Fraser, An optical coherence microscope for 3-dimensional imaging in developmental biology, Opt. Express 6, 136 (2000)

7. Y. Zhang, J. Rha, R.S. Jonnal, D.T. Miller, Adaptive optics parallel spectral domain optical coherence tomography for imaging the living retina, Opt. Express 13, 4792 (2005)

8. S. Bourquin, P. Seitz, R.P. Salathé, Optical coherence topography based on a twodimensional smart detector array, Opt. Lett. 26, 512 (2001)

9. E. Bordenave, E. Abraham, G. Jonusauskas, N. Tsurumachi, J. Oberlé, C. Rullière, P.E. Minot, M. Lassègues, J.E. Surlève Bazeille, Wide-field optical coherence tomography: imaging of biological tissues, Appl. Opt. 41, 2059 (2002)

10. M. Laubscher, M. Ducros, B. Karamata, T. Lasser, R. Salathé, Video-rate threedimensional optical coherence tomography, Opt. Express 10, 429 (2002)

11. B. Karamata, P. Lambelet, M. Laubscher, R.P. Salathé, T. Lasser, Spatially incoherent illumination as a mechanism for cross-talk suppression in wide-field optical coherence tomography, Opt. Lett. 29, 736 (2004)

12. L. Vabre, A. Dubois, A.C. Boccara, Thermal-light full-field optical coherence tomography, Opt. Lett., 27, 530 (2002)

13. LLTech SAS, France, http://www.1ltechimaging.com/

14. P. Xiao, M. Fink, A.C. Boccara, Full-field spatially incoherent illumination interferometry: a spatial resolution almost insensitive to aberrations, Opt. Lett. 41, 3920 (2016)

15. M. Born, E. Wolf, Principles of optics: electromagnetic theory of propagation, interference and diffraction of light (CUP Archive, 2000)

16. J.W. Goodman, Introduction to Fourier optics (Roberts and Company Publishers, 2005)

17. C.W. McCutchen, Generalized Source and the van Cittert-Zernike Theorem: A Study of the Spatial Coherence Required for Interferometry, J. Opt. Soc. Am. 56, 727 (1966)

18. H. Babcock, The possibility of compensating atmospheric seeing, Pub. Astr. Soc. Pac. 65, $229(2010)$ 
19. G. Rousset, J.C. Fontanella, P. Kern, F. Rigaut, First diffraction-limited astronomical images with adaptive optics, Astron. Astrophys. 230, L29 (1990)

20. J.A. Kubby, Adaptive Optics for Biological Imaging (CRC Press, 2013)

21. P. Godara, A.M. Dubis, A. Roorda, J.L. Duncan, J. Carroll, Adaptive optics retinal imaging: emerging clinical applications, Optom. Vis. Sci. 87, 930 (2010)

22. J. Porter, H.M. Queener, J.E. Lin, K. Thorn, A. Awwal (ed.), Adaptive Optics for Vision Science (John Wiley \& Sons, Inc., 2006)

23. D.R. Williams, Imaging single cells in the living retina, Vision Res. 51, 1379 (2011)

24. J. Liang, D.R. Williams, D.T. Miller, Supernormal vision and high-resolution retinal imaging through adaptive optics, J. Opt. Soc. Am. A 14, 2884 (1997)

25. A. Roorda, F. Romero-Borja, W. Donnelly III, H. Queener, T. Hebert, M. Campbell, Adaptive optics scanning laser ophthalmoscopy, Opt. Express 10, 405 (2002)

26. Y. Zhang, A Roorda, Evaluating the lateral resolution of the adaptive optics scanning laser ophthalmoscope, J. Biomed. Opt. 11, 14002 (2006)

27. D. Merino, J.L. Duncan, P. Tirubeedhula, A. Roorda, Observation of cone and rod photoreceptors in normal subjects and patients using a new generation adaptive optics scanning laser ophthalmoscope, Biomed. Opt. Express 2, 2189 (2011)

28. Y. Zhang, B. Cense, J. Rha, R.S. Jonnal, W. Gao, R.J. Zawadzki, J.S. Werner, S. Jone, S. Olivier, D.T. Miller, High-speed volumetric imaging of cone photoreceptoes with adaptive optics spectral-domain optical coherence tomography, Opt. Express 14, 4380 (2006)

29. R.J. Zawadzki, S.M. Jones, S.S. Olivier, M. Zhao, B.A. Bower, J.A. Izatt, S. Choi, S. Laut, J.S. Werner, Adaptive-optics optical coherence tomography for high-resolution and high-speed 3D retinal in vivo imaging, Opt. Express 13, 8532 (2005)

30. O.P. Kocaoglu, S. Lee, R.S. Jonnal, Q. Wang, A.E. Herde, J.C. Derby, W. Gao, D.T. Miller, Imaging cone photoreceptors in three dimensions and in time using ultrahigh resolution optical coherence tomography with adaptive optics, Biomed. Opt. Express 2, 748 (2011)

31. Y. Zhang, J. Rha, R. Jonnal, D. Miller, Adaptive optics parallel spectral domain optical coherence tomography for imaging the living retina, Opt. Express 13, 4792 (2005)

32. E.J. Fernández, B. Hermann, B. Povazay, A. Unterhuber, H. Sattmann, B. Hofer, P.K. Ahnelt, W. Drexler, Ultrahigh resolution optical coherence tomography and pancorrection for cellular imaging of the living human retina, Opt. Express 16, 11083 (2008)

33. E.J. Fernández, B. Povazay, B. Hermann, A. Unterhuber, H. Sattmann, P.M. Prieto, R. Leitgeb, P. Ahnelt, P. Artal, W. Drexler, Three-dimensional AO ultrahigh-resolution optical coherence tomography using a liquid crystal spatial light modulator, Vision Res. 45, $3432(2005)$

34. O.P. Kocaoglu, R.D. Ferguson, R.S. Jonnal, Z. Liu, Q. Wang, D.X. Hammer, D.T. Miller, Adaptive optics optical coherence tomography with dynamic retinal tracking, Biomed. Opt. Express 5, 2262 (2014)

35. K.S.K. Wong, Y. Jian, M. Cua, S. Bonora, R.J. Zawadzki, M.V. Sarunic, In vivo imaging of human photoreceptor mosaic with wavefront sensorless adaptive optics optical coherence tomography, Biomed. Opt. Express 6, 580 (2015)

36. M. Rueckel, J.A. Mack-Bucher, W. Denk, Adaptive wavefront correction in two-photon microscopy using coherence-gated wavefront sensing, PNAS 103, 17137 (2006)

37. S.H. Lee, J.S. Werner, R.J. Zawadzki, Improved visualization of outer retinal morphology with aberration cancelling reflective optical design for adaptive optics - optical coherence tomography, Biomed. Opt. Express 4, 2508 (2013)

38. J. Porter, A. Guirao, I.G. Cox, D.R. Williams, Monochromatic aberrations of the human eye in a large population, JOSA A 18, 1793 (2001)

39. J.F. Castejón-Mochón, N. López-Gil, A. Benito, P. Artal, Ocular wave-front aberration statistics in a normal young population, Vision Res. 42, 1611 (2002)

40. X. Hong, L. Thibos, A. Bradley, D. Miller, X. Cheng, N. Himebaugh, Statistics of aberrations among healthy young eyes, in Vision Science and its Applications, edited by A. Sawchuk, Vol. 53 of OSA Trends in Optics and Photonics (Optical Society of America, 2001), p. SuA5

41. D3128 Spatial Light Modulator, Meadowlark Optics, http://www.meadowlark.com/ 
42. G.D. Love, Wave-front correction and production of Zernike modes with a liquid-crystal spatial light modulator, Appl. Opt. 36, 1517 (1997)

43. A. Vyas, M.B. Roopashree, R.K. Banyal, B.R. Prasad, Spatial Light Modulator for wavefront correction, arXiv:0909.3413 (2009)

44. L.N. Thibos, A. Bradley, Use of liquid-crystal adaptive-optics to alter the refractive state of the eye, Optometry Vision Sci. 74, 581 (1997)

45. F. Vargas-Martın, P.M. Prieto, P. Artal, Correction of the aberrations in the human eye with a liquid-crystal spatial light modulator: limits to performance, JOSA A 15, 2552 (1998)

46. D.T. Miller, L.N. Thibos, X. Hong, Requirements for segmented correctors for diffraction-limited performance in the human eye, Opt. Express 13, 275 (2005)

47. P.N. Marsh, D. Burns, J.M. Girkin, Practical implementation of adaptive optics in multiphoton microscopy, Opt. Express 11, 1123 (2003)

48. L. Sherman, J.Y. Ye, O. Albert, T.B. Norris, Adaptive correction of depth-induced aberrations in multiphoton scanning microscopy using a deformable mirror, J. MicroscOxford 206, 65 (2002)

49. S. Zommer, E.N. Ribak, S.G. Lipson, J. Adler, Simulated annealing in ocular adaptive optics, Opt. Lett. 31, 939 (2006)

50. N. Ji, D.E. Milkie, E. Betzig, Adaptive optics via pupil segmentation for high-resolution imaging in biological tissues, Nat. Meth. 7, 141 (2009)

51. A. Dubois, G. Moneron, A.C. Boccara, Thermal-light full-field optical coherence tomography in the $1.2 \mu \mathrm{m}$ wavelength region. Opt. Commun. 266, 738 (2006)

52. S. Labiau, G. David, S. Gigan, A.C. Boccara, Defocus test and defocus correction in full-field optical coherence tomography, Opt. Lett. 34, 1576 (2009)

53. J. Binding, J.B. Arous, J. Leger, S. Gigan, C. Boccara, L. Bourdieu, Brian refractive index measured in vivo with high-NA defocus-corrected full-field OCT and consequences for two-photon microscopy, Opt. Express 19, 4833 (2011)

54. J. Wang, J. Leger, J. Binding, A.C. Boccara, S. Gigan, L. Bourdieu, Measuring aberrations on the rat brain by coherence-gated wavefront sensing using a Linnik interferometer, Biomed. Opt. Expess 3, 2510(2012)

55. P. Xiao, M. Fink, A.C. Boccara, Adapive optics full-field optical coherence tomography, submitted to Journal of Biomedical Optics

56. R.J. Noll, Zernike polynomials and atmospheric turbulence, JOsA 66, 207 (1976)

57. G. Dai, Wavefront Optics for Vision Correction (SPIE Press, 2008)

58. S. Bonora, R. J. Zawadzki, Wavefront sensorless modal deformable mirror correction in adaptive optics: optical coherence tomography, Opt. Lett. 38, 4801 (2013)

59. D. Debarre, M.J. Booth, T. Wilson, Image based adaptive optics through optimization of low spatial frequencies, Opt. Express 15, 8176 (2007)

60. J. Mertz, H. Paudel, T.G. Bifano, Field of view advantage of conjugate adaptive optics in microscopy applications, Appl. Opt. 54, 3498 (2015)

61. C.K. Hitzenberger, A. Baumgartner, W. Drexler, A.F. Fercher, Dispersion effects in partial coherence interferometry: implications for intraocular ranging, J. Biomed. Opt. 4, $144(1999)$

62. ROWE Technical Design, Inc., http://www.rowetechnical.com/tpme.html

Open Access This is an Open Access article distributed under the terms of the Creative Commons Attribution License (http://creativecommons.org/licenses/by/4.0), which permits unrestricted use, distribution, and reproduction in any medium, provided the original work is properly cited. 